\title{
The molecular infrastructure of glutamatergic synapses in the mammalian forebrain
}

J. Peukes ${ }^{1,2}$, M. Lovatt ${ }^{7}$, C. Leistner ${ }^{7}$, J. Boulanger ${ }^{1}$, D. R. Morado ${ }^{1,3}$, W. Kukulski1 ${ }^{1,4}$, F. Zhu $^{5,6}$, N. Komiyama $^{5,7}$, J. A. G. Briggs ${ }^{1}$, S. G. N. Grant ${ }^{5}$, R. Frank ${ }^{1,8^{*}}$

${ }^{1}$ MRC Laboratory of Molecular Biology, Francis Crick Avenue, Cambridge, CB2 0QH, UK.

${ }^{2}$ Structural and Computational Biology Unit, European Molecular Biology Laboratory (EMBL), 69117 Heidelberg, Germany.

${ }^{3}$ Current address: Science for Life Laboratory, Department of Biochemistry and Biophysics, Stockholm University, 17165 Solna, Sweden.

${ }^{4}$ Current address: Institute of Biochemistry and Molecular Medicine, University of Bern, 3012 Bern, Switzerland.

${ }^{5}$ Genes to Cognition Program, Centre for Clinical Brain Sciences, Simons Initiative for the Developing Brain, University of Edinburgh, Edinburgh EH16 4SB, UK.

${ }^{6}$ Current address: UCL Institute of Neurology, Queen Square, WC1N 3BG London, UK

${ }^{7}$ Genes to Cognition Program, Centre for Clinical Brain Sciences, Simons Initiative for the

Developing Brain, the Muir Maxwell Epilepsy Centre, University of Edinburgh, Edinburgh EH16 4SB, UK.

${ }^{8}$ Astbury Centre for Structural Molecular Biology, School of Biomedical Sciences, Faculty of Biological Sciences, University of Leeds, LS2 9JT, UK.

* Corresponding author. Email: r.frank@leeds.ac.uk

\begin{abstract}
Glutamatergic synapses form the vast majority of connections within neuronal circuits but how these subcellular structures are molecularly organized within the mammalian brain is poorly understood. Conventional electron microscopy using chemically fixed, metal-stained tissue has identified a proteinaceous, membrane-associated thickening called the 'postsynaptic density' (PSD). Here, we combined mouse genetics and cryo-electron tomography to determine the 3D molecular architecture of fresh synapses in the adult forebrain. The native glutamatergic synapse lacked a PSD. Instead, a concentrated 'synaptoplasm' consisting of cytoskeletal elements, macromolecular complexes and membrane-bound organelles extended throughout the pre- and post-synaptic compartments. Snapshots of active processes gave insights into the architectural basis for synaptic remodeling. Clusters of 4-60 ionotropic glutamate receptors were positioned inside and outside the synaptic cleft. Together, these information-rich tomographic maps present a detailed molecular framework for the coordinated activity within mammalian brain synapses.
\end{abstract}




\section{INTRODUCTION}

Glutamate is the major chemical neurotransmitter in the mammalian brain that mediates communication within neuronal circuits at specialized cell-cell junctions called synapses. The molecular composition of glutamatergic synapses is highly complex, reflecting the many different molecular machines required to subserve diverse synaptic functions, including learning and memory (1-4). Indeed, more than a thousand different synaptic proteins have been identified in the mouse and human forebrain $(5,6)$. How this ensemble of macromolecules within mammalian brain synapses is structurally organized is currently unknown.

Cryo-electron microscopy (cryoEM) has the potential to reveal the native 3-dimensional molecular architecture of mammalian brain synapses but with the limitation that samples must be vitreous and less than $\sim 300 \mathrm{~nm}$ thick. Although primary neurons grown on EM grids can overcome this constraint $(7,8)$, the environment in which in vitro cultured cells grow differs markedly from tissues. Indeed, primary neurons in vitro appear unable to reach the molecular and functional maturity of neurons from the brain itself $(2,9)$. While brain synapses can be isolated by biochemical fractionation, these enrichment steps delay cryopreservation by at least an hour and involve nonphysiological buffer that together cause deterioration of certain macromolecular structures. An example is the cytoskeleton, particularly microtubules, which rapidly depolymerize within a matter of 1-3 minutes if the provision of nucleotide triphosphates or cellular integrity becomes compromised $(10,11)$. To overcome these limitations, we developed an 'ultra-fresh' sample preparation workflow to examine mature mammalian brain synapses without fractionation by combining mouse genetics with cryogenic correlated light and electron microscopy (cryoCLEM) (Fig. 1A).

\section{RESULTS}

\section{CryoCLEM of adult brain glutamatergic synapses}

To determine the structure of native forebrain excitatory synapses we exploited knockin mice, in which the gene encoding PSD95 was engineered to include an in-frame, C-terminal fusion of the fluorescent tag, EGFP $\left(P s d 95^{G F P / G F P}\right)(2,12,13)$. PSD95 is a cytoplasmic protein that concentrates exclusively within mature glutamatergic synapses $(14,15)$ and forms supercomplexes with ionotropic glutamate receptors that reside in the postsynaptic membrane (2). Fresh $P s d 95^{G F P / G F P}$ mouse forebrains, encompassing cortex and hippocampus, were homogenized in ice-cold ACSF, 
then immediately vitrified on EM grids. Sample preparation was completed within 2 minutes, the time taken to cull the mouse, dissect, homogenize forebrain, and plunge-freeze the sample on a blotted cryoEM grid (Fig. 1A).

The GFP signal from $P s d 95^{G F P / G F P}$ knockin mice pinpoints the location of mature glutamatergic synapses without over-expression, or any detectible effect on synapse function (13). Thus, cryoEM grids were imaged on a cryogenic fluorescence microscope (cryoFM) revealing PSD95-GFP puncta (Fig. 1B), absent in WT control samples (Fig. S1A), which resembled those observed by confocal fluorescent imaging of intact tissues (12). Using a cryoCLEM workflow the same grids were then imaged by cryoEM and fluorescent PSD95-GFP puncta were mapped onto the cryoEM image (Fig. 1B). These cryoCLEM images showed PSD95-GFP puncta that were invariably associated with membrane-bound subcellular compartments (100 to 1,300 nm diameter) (Fig. 1B).

To resolve the molecular architecture within PSD95-containing puncta, we acquired 93 PSD95GFP cryoCLEM-guided cryo-electron tomographic tilt series from 5 adult mouse forebrains. Aligned tilt series were reconstructed to produce 3-dimensional tomographic maps as shown in Figure 1C and D (all tomograms available in the EMPIAR database upon publication). As expected, all GFP-positive membranes were attached to a neighbouring membrane, of which $78 \%$ were closed compartments and enriched in synaptic vesicles (15-35 nm diameter) that characterize the presynaptic compartment. Thus, our PSD95-GFP cryoCLEM workflow enabled unambiguous identification of the pre- and post-synaptic membrane of glutamatergic synapses (hereon referred to as PreSM and PoSM, respectively).

The high quality of the tomographic maps enabled assignment of cytoskeletal elements within the synapse, including extensive networks of filamentous actin (F-actin). These were identifiable with a diameter of $7 \mathrm{~nm}$ and apparent helical arrangement of subunits (16) in all PoSM compartments (Fig. 1C, D, E and Fig. S1B) and in 49 $\pm 7 \%$ (mean \pm SEM, $n=5$ mice) of PreSM compartments (Fig. $1 \mathrm{E}$ and S1C). Microtubules were identified (25 nm diameter) in $15 \pm 2 \%$ of PreSM (Fig. 1C, D, and F), but as expected were absent from the PoSM (17). Notwithstanding the mechanical stress of detaching synaptic terminals to cryopreserve whole synapses, this extensive actin and microtubular cytoskeleton corroborates the maintenance of native macromolecular structures.

\section{Molecular crowding within the synapse}

We first assessed the overall architecture of macromolecules within the synapse by measuring 
macromolecular crowding. Based on conventional EM experiments of fixed, dehydrated heavymetal-stained tissue, glutamatergic synapses have been characterised by the presence of a canonical 30-50 $\mathrm{nm}$ layer of densely packed proteins on the cytoplasmic side of the postsynaptic membrane, called the postsynaptic density (PSD) $(18,19)$. However, within fresh, native brain synapse structures reported here no such postsynaptic density was apparent (Fig. 1C, D). Indeed, the average density of the cytoplasm was as high within proximal regions near to the postsynaptic membrane as it was within distal regions several hundred nanometers away (Fig. 2A). Moreover, molecular crowding was equally high in the presynaptic compartment (Fig. 2A). Therefore, it is likely the PSD observed in conventional EM misrepresents the organization of synapses and arises because the cytoplasm of the synapse is washed away or denatured leaving the remnants of only a fraction of the proteins that are crosslinked to the postsynaptic membrane.

To test this hypothesis, we cryopreserved acute slice preparations of anatomically intact adult $P s d 95^{G F P / G F P}$ forebrain by high-pressure freezing and collected $150 \mathrm{~nm}$ thin, vitreous sections by cryo-ultramicrotomy (Fig. 2B) (20). Glutamatergic synapses were identified within these fresh tissue cryo-sections by cryoCLEM, from which cryo-electron tomograms were collected revealing the native in-tissue architecture of glutamatergic synapses (Fig. 2C). Molecular crowding profiles of these synapses confirmed the absence of a PSD (Fig. 2D) and were consistent with those observed in isolated 'ultra-fresh' samples (Fig. 2A) and previously reported unlabeled synapses in cultured immature brain slices (20). Thus, instead of a PSD, the native architecture of mature glutamatergic brain synapse reveals a 'synaptoplasm' consisting of an ornate, branched network of cytoskeletal elements, organelles and macromolecular complexes that extends throughout the postsynaptic compartment.

\section{Synaptic organelles}

To assess the diversity of architectures we next catalogued the identifiable macromolecular and organelle constituents in ultra-fresh synapses (Table S1). At least one membrane-bound organelle was present in $61 \pm 4 \%$ of PoSM compartments and, excluding synaptic vesicles, in $77 \pm 2 \%$ of PreSM compartments (Fig. 2E). These were readily separable into four structural classes on the basis of membrane protein composition, curvature and size (Fig. 2F and Table S1, see methods): 1) A network of flat, tubular membrane compartments that twisted and projected deep toward the adhered synaptic membrane (Fig. 2F and G, Fig. S2A). 2) Large spheroidal membrane compartments ( $>60 \mathrm{~nm}$ diameter) that were situated $72-500 \mathrm{~nm}$ away from the cleft (Fig. $2 \mathrm{~F}$ and G, Fig. S2B). 3) Multivesicular bodies that were positioned throughout the PreSM and PoSM (78- 
$360 \mathrm{~nm}$ away from the cleft, Fig. 2F and H, Fig. S2C). 4) Polyhedral vesicles with flat surfaces and joined by protein-coated membrane edges and vertices $\left(90^{\circ}\right.$ to $35^{\circ}$ internal angle, Fig. $2 \mathrm{~F}$ and I, Fig. S2D). Except for mitochondrial cristae, internal membranes with this high curvature have not been reported in cryo-electron tomograms of non-neuronal cells $(21,22)$.

Mitochondria were also readily identified, most abundantly in the presynaptic compartment (44 $\pm 5 \%$ of PreSM) and only once in close proximity to the postsynaptic compartment (Fig. S3A). A striking feature of all presynaptic mitochondria was the presence of amorphous dense aggregates within the mitochondrial matrix (Fig. S3B). Similar aggregates have been characterized in cultured cells and identified as solid deposits of calcium phosphate (23). Thus, mitochondrial granular aggregates likely represent a physiologically normal feature of the mammalian brain, which is in keeping with the role of synaptic mitochondria as a reservoir of cellular calcium ions (24). Overall, the variable protein composition and type of intracellular organelles indicate distinct synapse subtypes or states within the mammalian forebrain.

\section{Synaptic shape and remodelling}

Further diversity of synaptic architectures was evident in the size, shape and mode of connectivity of the PreSM to the PoSM (12). To assess these structural variables, PSD95-GFP-containing PoSMs and apposing PreSMs were semi-automatically segmented (see methods). This revealed synapses with multiple different topologies, including with a single, double, and divergent (1 PreSM: 2 PoSM) synaptic contact (Fig 3A, top, middle and bottom, respectively). These are consistent with the known diversity of cellular connectivity mediated by glutamatergic synapses (25). The volume of the PoSM compartment in our dataset ranged from 3 to $79 \times 10^{6} \mathrm{~nm}^{3}$. PoSM size was not correlated with the amount of PSD95-GFP detected by cryoCLEM ( $\mathrm{P}=0.7$, Pearson's $\mathrm{r}=0.06$, Fig. 3B). However, PoSM size was correlated with the number of F-actin cytoskeletal filaments $(\mathrm{P}<0.0001$, Pearson's r=0.83, Fig. $3 \mathrm{C})$, which is in line with the role of F-actin in regulating synaptic geometry and structural plasticity (17).

The variation in size, shape and molecular composition of synaptic membranes is likely to arise by synaptic remodeling. In $32 \pm 6 \%$ synapses we identified intermediates of membrane remodeling (Fig. 3D) that were trapped within the sample at the moment of freezing (Table S1). Significantly more remodelling intermediates were identified in the PreSM compared to PoSM compartments (Fig. $3 \mathrm{E})$, which is consistent with the expected high frequency of membrane dynamics associated with synaptic vesicle recycling in this compartment (26). 
In a subset of tomograms $(21 \pm 11 \%)$ a clathrin coat was unambiguously identified (Fig. F) by the triskelion-forming pentagonal and hexagonal openings (27) evident in the raw tomographic maps (Table S1). In the PreSM, clathrin surrounded synaptic vesicles (Fig. 3G) and budding vesicles on internal membrane compartments within the PreSM (Fig. 3H), which is in agreement with previous time-resolved EM experiments (28). In the postsynaptic compartment, a clathrin-coated pit formed directly on the cleft, suggesting membrane and cargo can be removed without prior lateral diffusion out of the synapse (Fig. 3I). Thus, the distribution of these distinct intermediates suggests that membrane remodelling occurs at particular sub-compartments of the synapse $(28,29)$.

\section{Synaptic cleft and ionotropic glutamate receptors}

Geometric properties of each synapse are thought to be critical for determining synaptic strength, including synaptic cleft height (the distance between PreSM and PoSM) $(30,31)$. To quantify this variable in our dataset we computationally determined the 3D coordinates of the cleft within each synapse (Fig. S4A and see methods). The PreSM to PoSM distances across the cleft indicated an average cleft height of $33 \mathrm{~nm}$ (Fig. 4A). These values differ significantly from cleft heights of $\sim 20$ $\mathrm{nm}$ determined in non-native conditions by conventional EM (32) but are consistent with superresolution light microscopy imaging (33). Interestingly, the average cleft height of each synapse in our dataset varied (ranging from 27 to $37 \mathrm{~nm}$ ) and a subset of synapses maintained a very broad or bimodal distribution of cleft heights (Fig. S4B). Inspection of synapses with a broad or bimodal distribution revealed closely apposed and remotely spaced subsynaptic regions of the cleft, containing transsynaptic complexes with varying dimensions that complemented the cleft height of the subregion (Fig. S4C). Thus, these data suggest the synaptic cleft as a highly plastic interface, where cleft dimensions are dictated by the variable molecular composition of transsynaptic adhesion complexes (34).

The number, concentration, and distribution of ionotropic glutamate receptors anchored at the postsynaptic membrane by PSD95 is a central determinant of synaptic output (30, 31, 35-38). Putative ionotropic glutamate receptors were readily identified in $95 \pm 2 \%$ of PoSMs. These resembled the side (Fig. 4B left) and top views (Fig. 4B right) of ionotropic glutamate receptor atomic models (39-41). To confirm the identity of these proteins, we manually picked 2,522 receptors and extracted sub-volumes at those positions. We then applied subtomogram averaging procedures (see methods) which gave a $25 \AA$ resolution ' $Y$ ' shaped cryoEM density map extending 
$14 \mathrm{~nm}$ from the PoSM (Fig. S5A). The density shows a 2-fold symmetric structure containing two layers of globular domains in positions proximal and distal to the PoSM (Fig. 4C) as expected for the structure of an ionotropic glutamate receptor. Consequently, the atomic structure of the AMPA receptor (42) was well accommodated by the density, which validates the identification of these ion channels within our synapse tomogram dataset (Fig. 4C).

Next, to quantify the 3D configuration of populations of ionotropic glutamate receptors, we used these coordinates to identify clusters of receptors (see methods). Clusters were identified in $78 \%$ of synapses with an average of 2 clusters per synapse (Fig. 4D), which were composed of 4 to 60 ionotropic glutamate receptors (average 10 receptors per cluster) (Fig. 4E). These subsynaptic regions with higher concentrations of ionotropic glutamate receptors were reminiscent of receptor 'nanodomains' detected by super-resolution microscopy $(13,36,43-45)$.

The position of ionotropic receptor clusters in synaptic and extra-synaptic sites, particularly the most abundant AMPA receptor subtype, is expected to impact synaptic strength and play an important role in synaptic plasticity (46). We therefore analyzed the distribution of clusters in the context of the overall synapse architecture, which revealed that $60 \%$ of synapses maintained multiple large clusters of ionotropic glutamate receptors completely or partly outside of the cleft (Fig. 4D and F). The detection of perisynaptic populations of glutamate receptors is consistent with previous freeze-fracture immunogold EM (47) and super-resolution light microscopy (44). Indeed, a population of ionotropic glutamate receptors outside the cleft is functionally necessary for the rapid reorganization of receptors during the early phase of long-term potentiation (48). Interestingly, the cytoplasmic C-termini of ionotropic receptor clusters both inside and outside of the cleft were associated with an elaborate and heterogeneous membrane- and actin-associated machinery (Fig. $\mathrm{S} 5 \mathrm{~B})$, possibly indicating that a structural mechanism contributes to the positioning of receptors both inside and outside the synaptic cleft.

\section{Conclusion}

Overall, the apparent variability of molecular and membrane architectures is in keeping with the enormous diversity of glutamatergic synapse types in the mammalian brain (12). Notwithstanding the absence of the PSD, the arrangement of prominent constituents, including branched F-actin networks, organelles, ionotropic glutamate receptors and transsynaptic adhesion complexes, appeared to define the architecture of each glutamatergic synapse. A future challenge will be to 
decipher how synaptic structural variability across the dendritic tree, different brain regions and neuronal subtypes determine specific functions within the mammalian brain. 


\section{References}

1. Y. Nakahata, R. Yasuda, Plasticity of Spine Structure: Local Signaling, Translation and Cytoskeletal Reorganization. Front. Synaptic Neurosci. 10, 1194-13 (2018).

2. R. A. W. Frank et al., NMDA receptors are selectively partitioned into complexes and supercomplexes during synapse maturation. Nature Communications. 7, 11264 (2016).

3. S. Ebrahimi, S. Okabe, Structural dynamics of dendritic spines: Molecular composition, geometry and functional regulation. BBA - Biomembranes. 1838, 2391-2398 (2014).

4. J. J. E. Chua, S. Kindler, J. Boyken, R. Jahn, The architecture of an excitatory synapse. $J$ Cell Sci. 123, 819-823 (2010).

5. A. Bayés et al., Characterization of the proteome, diseases and evolution of the human postsynaptic density. Nature neuroscience. 14, 19-21 (2011).

6. M. Roy et al., Proteomic analysis of postsynaptic proteins in regions of the human neocortex. Nature neuroscience. 21, 130-138 (2017).

7. S. Asano et al., A molecular census of $26 \mathrm{~S}$ proteasomes in intact neurons. Science. 347, 439-442 (2015).

8. C.-L. Tao et al., Differentiation and Characterization of Excitatory and Inhibitory Synapses by Cryo-electron Tomography and Correlative Microscopy. J Neurosci. 38, 1493-1510 (2018).

9. A. Z. Harris, D. L. Pettit, Extrasynaptic and synaptic NMDA receptors form stable and uniform pools in rat hippocampal slices. J Physiol (Lond). 584, 509-519 (2007).

10. T. D. Pollard, The role of actin in the temperature-dependent gelation and contraction of extracts of Acanthamoeba. J Cell Biol. 68, 579-601 (1976).

11. T. J. Mitchison, Evolution of a dynamic cytoskeleton. Philos Trans R Soc Lond, B, Biol Sci. 349, 299-304 (1995).

12. F. Zhu et al., Architecture of the Mouse Brain Synaptome. Neuron. 99, 781-799 (2018).

13. M. J. Broadhead et al., PSD95 nanoclusters are postsynaptic building blocks in hippocampus circuits. Sci. Rep. 6, 1-14 (2016).

14. A. E. El-Husseini, E. Schnell, D. M. Chetkovich, R. A. Nicoll, D. S. Bredt, PSD-95 involvement in maturation of excitatory synapses. Science. 290, 1364-1368 (2000).

15. L. Chen et al., Stargazin regulates synaptic targeting of AMPA receptors by two distinct mechanisms. Nature. 408, 936-943 (2000).

16. J. Hanson, Axial Period of Actin Filaments. Nature. 213, 353-356 (1967). 
17. K. Okamoto, M. Bosch, Y. Hayashi, The Roles of CaMKII and F-Actin in the Structural Plasticity of Dendritic Spines: A Potential Molecular Identity of a Synaptic Tag? Physiology. 24, 357-366 (2009).

18. M. Sheng, E. Kim, The Postsynaptic Organization of Synapses. Cold Spring Harbor Perspectives in Biology. 3, 1-21 (2011).

19. E. G. Gray, Axo-somatic and axo-dendritic synapses of the cerebral cortex: An electron microscope study. Journal of Anatomy. 93, 420-433 (1959).

20. B. Zuber, I. Nikonenko, P. Klauser, D. Muller, J. Dubochet, The mammalian central nervous synaptic cleft contains a high density of periodically organized complexes. $P$ Natl Acad Sci Usa. 102, 19192-19197 (2005).

21. B. D. Engel et al., In situ structural analysis of Golgi intracisternal protein arrays. $P$ Natl Acad Sci Usa. 112, 11264-11269 (2015).

22. Y. S. Bykov et al., The structure of the COPI coat determined within the cell. eLife. 6 , 6916 (2017).

23. S. G. Wolf et al., 3D visualization of mitochondrial solid-phase calcium stores in whole cells. eLife. 6, 147 (2017).

24. B. Billups, I. D. Forsythe, Presynaptic mitochondrial calcium sequestration influences transmission at mammalian central synapses. Journal of Neuroscience. 22, 5840-5847 (2002).

25. K. M. Harris, R. J. Weinberg, Ultrastructure of Synapses in the Mammalian Brain. Cold Spring Harbor Perspectives in Biology. 4, a005587-a005587 (2012).

26. N. L. Kononenko, V. Haucke, Molecular Mechanisms of Presynaptic Membrane Retrieval and Synaptic Vesicle Reformation. Neuron. 85, 484-496 (2015).

27. T. Kanaseki, THE "VESICLE IN A BASKET": A Morphological Study of the Coated Vesicle Isolated from the Nerve Endings of the Guinea Pig Brain, with Special Reference to the Mechanism of Membrane Movements. J Cell Biol. 42, 202-220 (1969).

28. S. Watanabe et al., Clathrin regenerates synaptic vesicles from endosomes. Nature. 515, 228-233 (2014).

29. C. Imig et al., Ultrastructural Imaging of Activity-Dependent Synaptic MembraneTrafficking Events in Cultured Brain Slices. Neuron. 108, 843-860.e8 (2020).

30. D. A. Rusakov, L. P. Savtchenko, K. Zheng, J. M. Henley, Shaping the synaptic signal: molecular mobility inside and outside the cleft. Trends Neurosci. 34, 359-369 (2011).

31. L. Cathala, N. B. Holderith, Z. Nusser, D. A. DiGregorio, S. G. Cull-Candy, Changes in synaptic structure underlie the developmental speeding of AMPA receptor-mediated EPSCs. Nature neuroscience. 8, 1310-1318 (2005). 
32. A. Peters, S. L. PALAY, H. F. Webster, The fine structure of the nervous system: the cells and their processes (1970).

33. J. Tønnesen, V. V. G. K. Inavalli, U. V. Nägerl, Super-Resolution Imaging of the Extracellular Space in Living Brain Tissue. Cell. 172, 1108-1111.e15 (2018).

34. M. Missler, T. C. Sudhof, T. Biederer, Synaptic Cell Adhesion. Cold Spring Harbor Perspectives in Biology. 4, a005694-a005694 (2012).

35. J. M. Kerr, T. A. Blanpied, Subsynaptic AMPA Receptor Distribution Is Acutely Regulated by Actin-Driven Reorganization of the Postsynaptic Density. J Neurosci. 32, 658-673 (2012).

36. A.-H. Tang et al., A trans-synaptic nanocolumn aligns neurotransmitter release to receptors. Nature. 536, 210-214 (2016).

37. J.-C. Beique et al., Synapse-specific regulation of AMPA receptor function by PSD-95. Proceedings of the National Academy of Sciences. 103, 19535-19540 (2006).

38. M. Migaud et al., Enhanced long-term potentiation and impaired learning in mice with mutant postsynaptic density-95 protein. Nature. 396, 433-439 (1998).

39. I. H. Greger, M. L. Mayer, Structural biology of glutamate receptor ion channels: towards an understanding of mechanism. Current Opinion in Structural Biology. 57, 185-195 (2019).

40. S. Zhu, E. Gouaux, Structure and symmetry inform gating principles of ionotropic glutamate receptors. Neuropharmacology. 112, 11-15 (2017).

41. M. C. Regan, A. Romero-Hernandez, H. Furukawa, A structural biology perspective on NMDA receptor pharmacology and function. Current Opinion in Structural Biology. 33, 68-75 (2015).

42. A. I. Sobolevsky, M. P. Rosconi, E. Gouaux, X-ray structure, symmetry and mechanism of an AMPA-subtype glutamate receptor. Nature. 462, 745-756 (2009).

43. A. Dani, B. Huang, J. Bergan, C. Dulac, X. Zhuang, Superresolution imaging of chemical synapses in the brain. Neuron. 68, 843-856 (2010).

44. D. Nair et al., Super-resolution imaging reveals that AMPA receptors inside synapses are dynamically organized in nanodomains regulated by PSD95. Journal of Neuroscience. 33, 13204-13224 (2013).

45. H. D. MacGillavry, Y. Song, S. Raghavachari, T. A. Blanpied, Nanoscale Scaffolding Domains within the Postsynaptic Density Concentrate Synaptic AMPA Receptors. Neuron. 78, 615-622 (2013).

46. M. Park, AMPA Receptor Trafficking for Postsynaptic Potentiation. Front. Cell. Neurosci. 12, 59-10 (2018). 
47. M. Masugi-Tokita, R. Shigemoto, High-resolution quantitative visualization of glutamate and GABA receptors at central synapses. Curr Opin Neurobiol. 17, 387-393 (2007).

48. A. C. Penn et al., Hippocampal LTP and contextual learning require surface diffusion of AMPA receptors. Nature. 549, 384-388 (2017).

49. C. J. Russo, S. Scotcher, M. Kyte, A precision cryostat design for manual and semiautomated cryo-plunge instruments. Review of Scientific Instruments. 87, 114302-6 (2016).

50. W. J. H. Hagen, W. Wan, J. A. G. Briggs, Implementation of a cryo-electron tomography tilt-scheme optimized for high resolution subtomogram averaging. Journal of Structural Biology. 197, 191-198 (2017).

51. D. N. Mastronarde, Automated electron microscope tomography using robust prediction of specimen movements. Journal of Structural Biology. 152, 36-51 (2005).

52. Y. Fukuda, U. Laugks, V. Lucic, W. Baumeister, R. Danev, Electron cryotomography of vitrified cells with a Volta phase plate. Journal of Structural Biology. 190, 143-154 (2015).

53. M. Schorb, J. A. G. Briggs, Correlated cryo-fluorescence and cryo-electron microscopy with high spatial precision and improved sensitivity. Ultramicroscopy. 143, 24-32 (2014).

54. W. Kukulski et al., Correlated fluorescence and 3D electron microscopy with high sensitivity and spatial precision. J Cell Biol. 192, 111-119 (2011).

55. J. R. Kremer, D. N. Mastronarde, J. R. McIntosh, Computer Visualization of ThreeDimensional Image Data Using IMOD. Journal of Structural Biology. 116, 71-76 (1996).

56. T. Wagner et al., SPHIRE-crYOLO is a fast and accurate fully automated particle picker for cryo-EM. Communications Biology, 1-13 (2019).

57. D. Castaño-Díez, M. Kudryashev, H. Stahlberg, Dynamo Catalogue: Geometrical tools and data management for particle picking in subtomogram averaging of cryo-electron tomograms. Journal of Structural Biology. 197, 135-144 (2017).

58. S. Nickell et al., TOM software toolbox: acquisition and analysis for electron tomography. Journal of Structural Biology. 149, 227-234 (2005).

59. F. Förster, O. Medalia, N. Zauberman, W. Baumeister, D. Fass, Retrovirus envelope protein complex structure in situ studied by cryo-electron tomography. P Natl Acad Sci Usa. 102, 4729-4734 (2005).

60. W. Wan et al., Structure and assembly of the Ebola virus nucleocapsid. Nature. 51, 394397 (2017).

61. S. Chen et al., High-resolution noise substitution to measure overfitting and validate resolution in 3D structure determination by single particle electron cryomicroscopy. Ultramicroscopy. 135, 24-35 (2013). 
62. E. F. Pettersen et al., UCSF Chimera - A visualization system for exploratory research and analysis. J. Comput. Chem. 25, 1605-1612 (2004).

\section{Acknowledgements}

We would like to thank Christos Savvas, Giuseppe Cannone and Shaoxia Chen for help with maintaining and setting up MRC LMB Titan Krios microscopes. We would like to thank the Astbury Biostructure Laboratory (ABSL), particularly Rebecca Thompson, Emma Hesketh, Dan Maskell, Martin Fuller and Helmut Gnaegi for help with setting up and maintaining HPF, cryoultramicrotome, cryoFM and Titan Krios microscopes. We are grateful to Jake Grimmett and Toby Darling for providing essential computational support. Heather Lloyd and Biomedical staff for technical support. Madeleine Gilbert and Eva Martínez Barceló for assistance with annotation of synapse tomograms. Grant funding: An Academy of Medical Sciences Springboard Award and a University of Leeds Academic Fellowship to RAWF. A Wellcome Trust (Technology Development Grant 202932), the European Research Council (ERC) under the European Union's Horizon 2020 Research and Innovation Programme (695568 SYNNOVATE), Simons Foundation for Autism Research Initiative (529085) to SGGN and NHK. Medical Research Council (MC_UP_1201/16) and the European Research Council (ERC) under the European Union's Horizon 2020 research and innovation programme (ERC-CoG-648432 MEMBRANEFUSION) to JAGB. WK was supported by the Medical Research Council (MC_UP_1201/8). ABSL Titan Krios microscopes were funded by the University of Leeds and Wellcome Trust (108466/Z/15/Z). The Leica EM ICE, UC7 ultra/cryo-ultramicrotome and cryoCLEM systems were funded by Wellcome Trust (208395/Z/17/Z) 


\section{Figure 1}

A

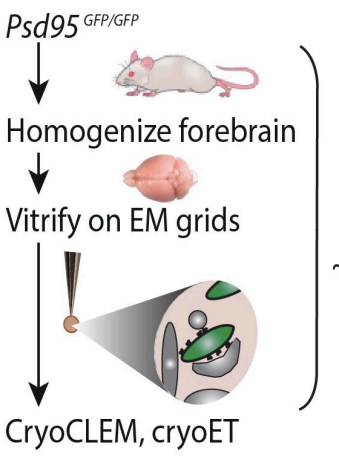

B

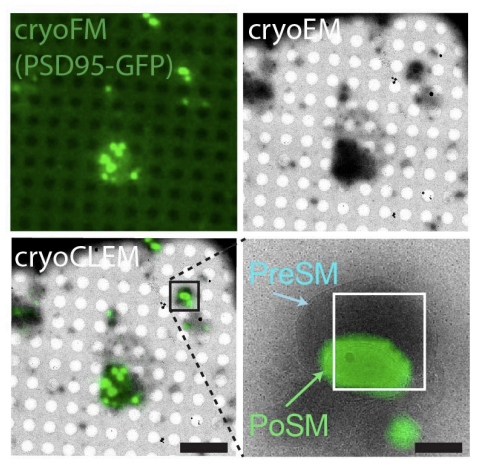

D

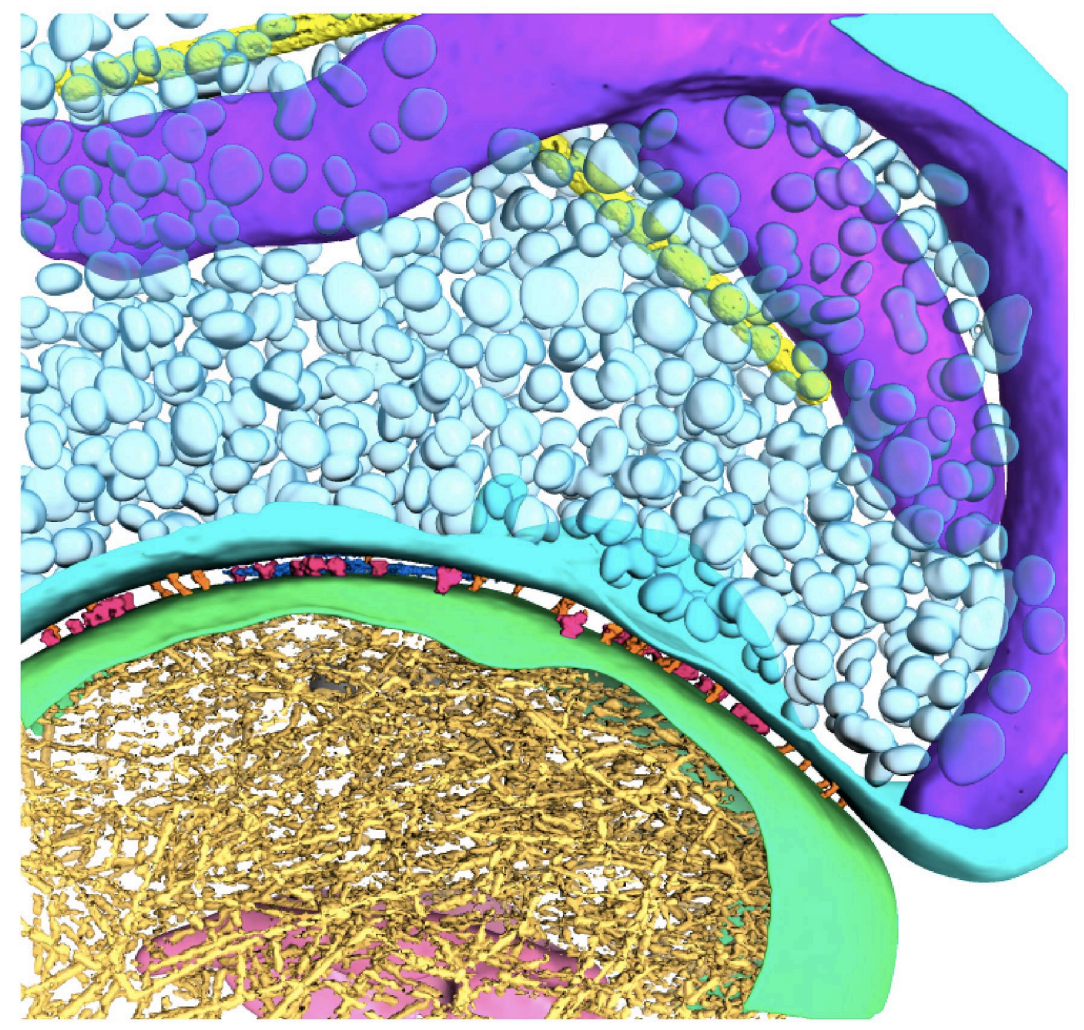

\section{C}

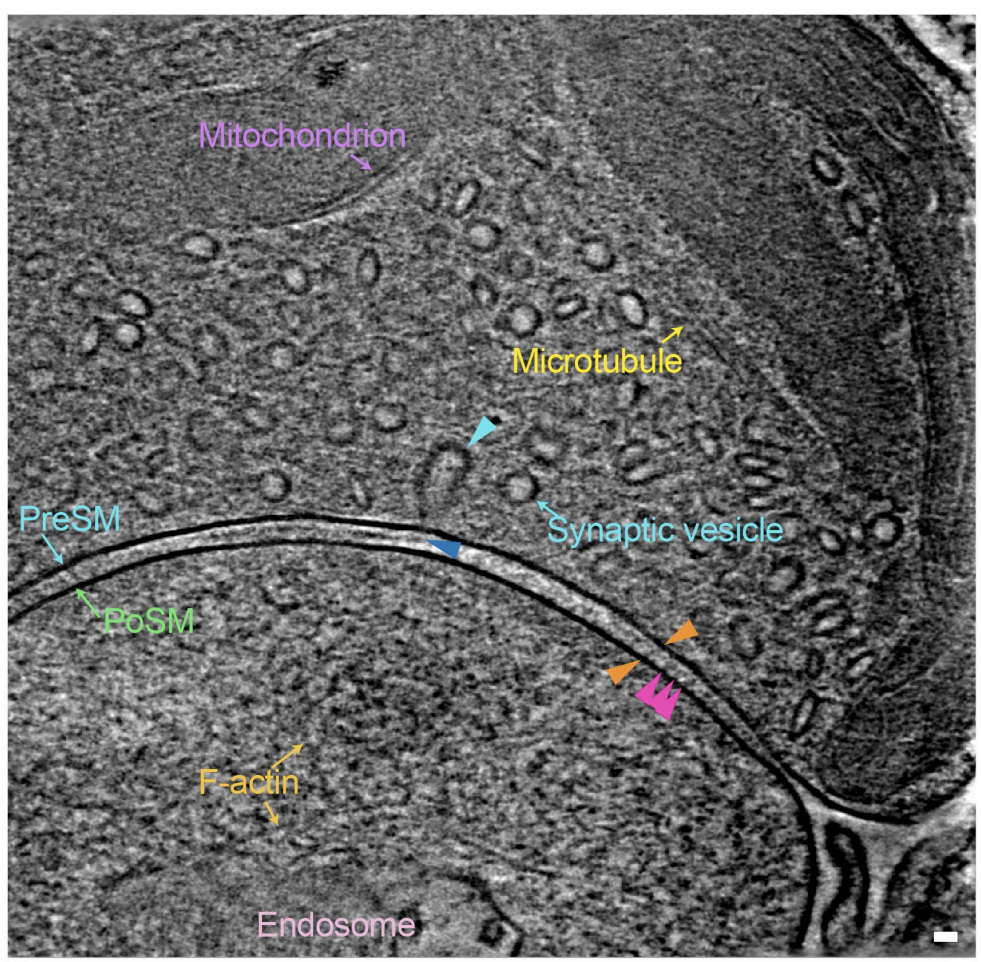

E

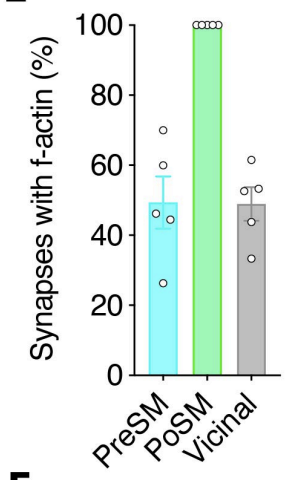

$F$

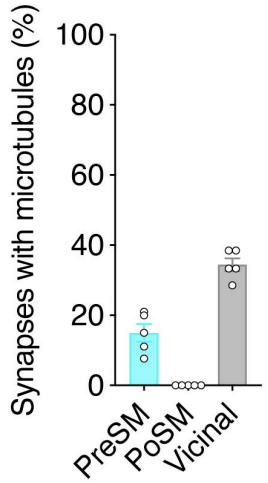


Figure 1. CryoCLEM and cryoET of mouse forebrain synapses.

(A) Schematic of the 'ultra-fresh' preparation of synapses for cryoCLEM. Psd95 ${ }^{G F P / G F P}$ knockin mice are culled, forebrain is dissected, homogenized in ice cold artificial cerebrospinal fluid, and plunge-frozen on cryoEM grids. 2 minutes is the time taken to cull, dissect and cryopreserve samples on EM grids.

(B) CryoCLEM of cryoEM grid square containing an ultra-fresh synapse preparation. Top left, cryoFM image of a holey carbon grid square. Top right, cryoEM image of the same grid square shown in top left. Bottom left, merged image of cryoFM and cryoEM images indicating location of PSD95-GFP puncta. Scale bar, $5 \mu \mathrm{m}$. Black box indicates region enlarged in Bottom right, showing PSD95-GFP associated with PoSM. White box indicates region where images for the tomogram shown in $\mathrm{C}$ were acquired. Scale bar, $500 \mathrm{~nm}$.

(C) Virtual slice through tomographic map of PSD95-contaning glutamatergic synapse. The PoSM (green) was identified by PSD-GFP cryoCLEM and the PreSM (cyan) was identified by its tethering to the PoSM and the prevalence of synaptic vesicles. Salient organelle and macromolecular constituents are indicated: Purple arrow, mitochondrion. Yellow arrow, microtubule. Cyan arrow, synaptic vesicle and cyan arrowhead indicating intermediate of vesicle fission/fusion. Gold arrow, F-actin filament. Pink arrow, putative endosomal compartment. Orange arrowheads, transsynaptic macromolecular complex bridging PreSM and PoSM. Magenta arrowheads, postsynaptic membrane proteins with extracellular domains extending 13-14 nm into the synaptic cleft. Blue arrow, lateral matrix of macromolecules in synaptic cleft. Scale bar, $20 \mathrm{~nm}$. The tomographic map shown here is representative of 93 tomograms obtained across 5 mouse forebrain preparations.

(D) 3D segmentation of membranes and macromolecules in a representative tomographic volume of a PSD95-containing glutamatergic synapse. Coloured as in C.

(E) Prevalence of branched filamentous actin networks in presynaptic membrane compartments (PreSM, cyan), PSD95-containing postsynaptic membrane compartments (PoSM, green), and neighbouring non-synaptic membrane compartments (Vicinal, grey). Data points are per mouse, for 5 adult $P s d 95^{G F P / G F P}$ mouse forebrain samples. Error bars, SEM.

(F) Same as E but for microtubules. 
bioRxiv preprint doi: https://doi.org/10.1101/2021.02.19.432002; this version posted February 19, 2021. The copyright holder for this preprint (which was not certified by peer review) is the author/funder. All rights reserved. No reuse allowed without permission.

Figure 2

A

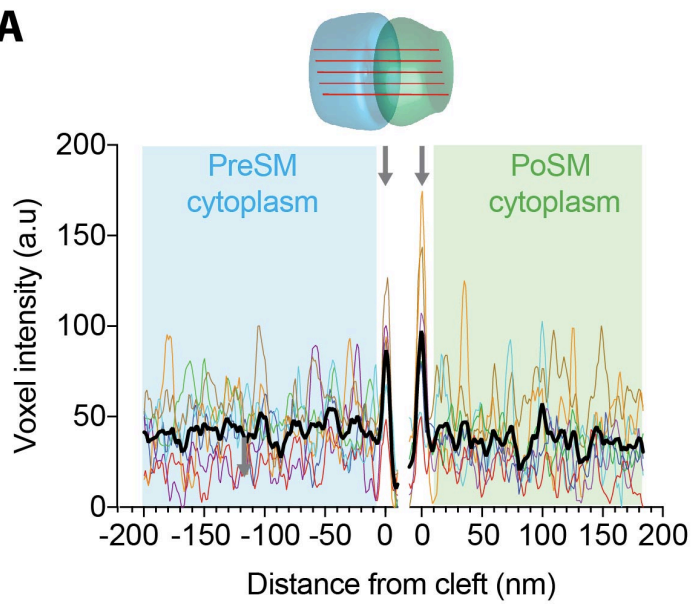

D

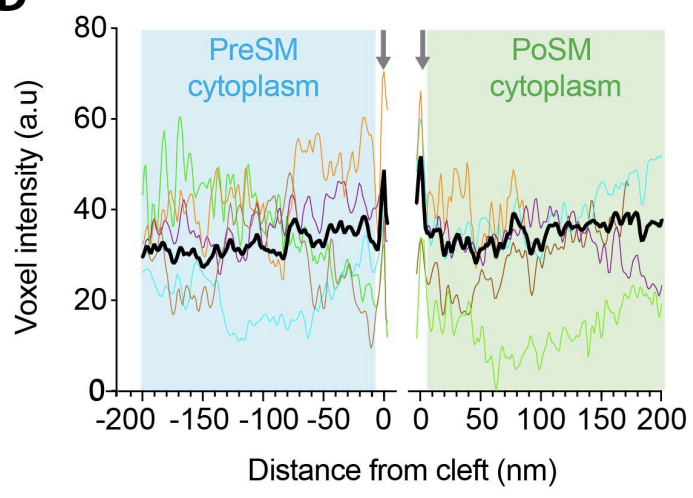

B C

PSd95 GFP/GFP
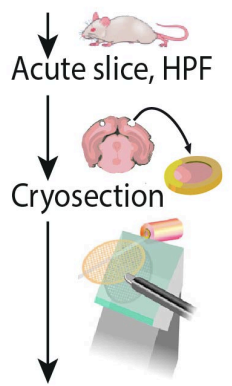

CryoCLEM, Cryo-ET

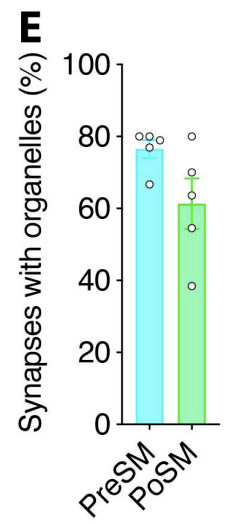

F
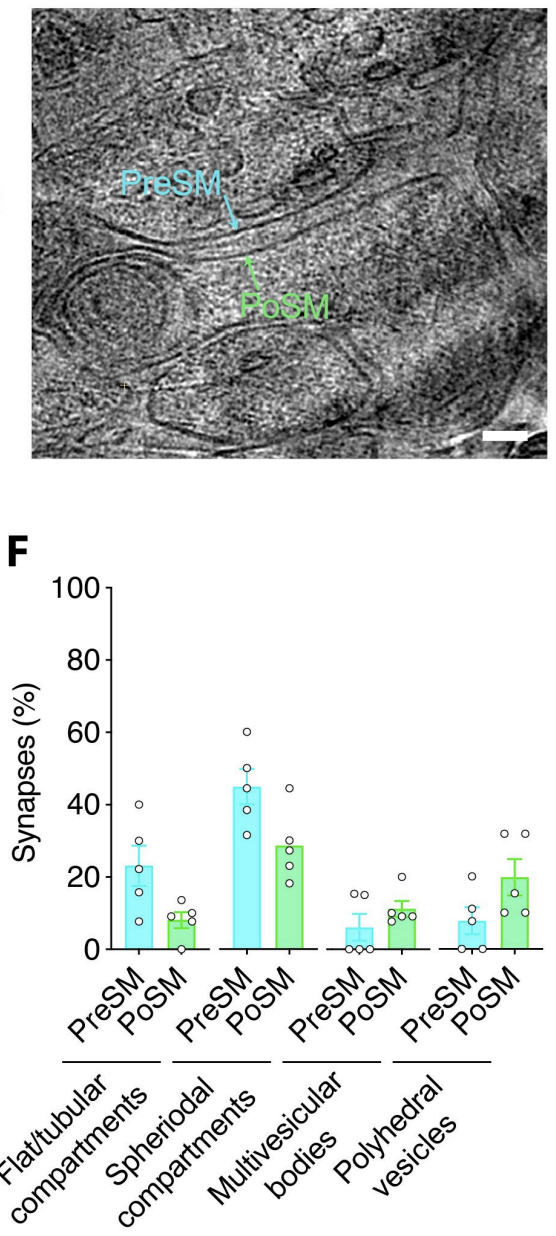

G
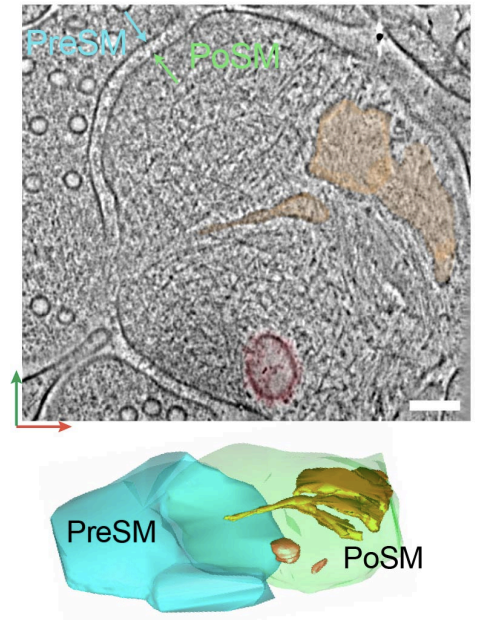

H
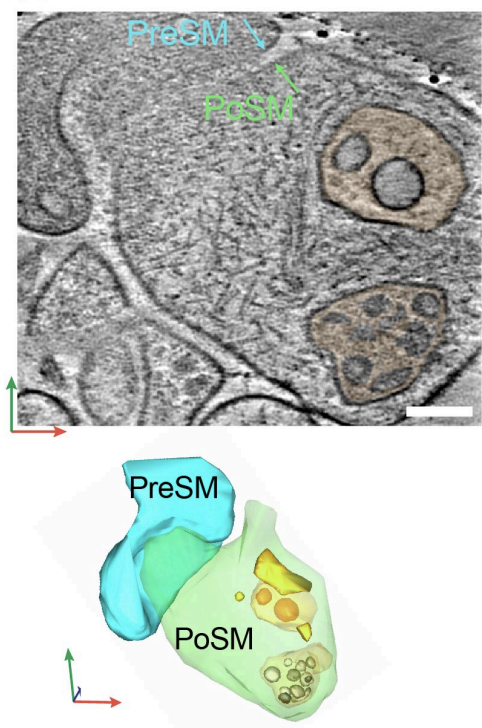

I
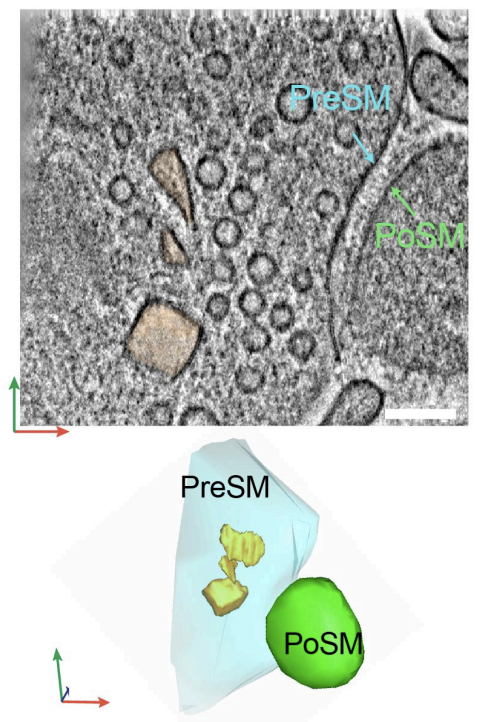
Figure 2. The architecture of native glutamatergic synaptic cytoplasm.

(A) Molecular crowding of the PreSM and PoSM cytoplasm in ultra-fresh synapse preparation. Top, schematic showing the measurement of molecular density estimated with multiple line profiles plots of voxel intensity within each synapse tomogram. Bottom, voxel intensity (a.u., arbitrary units) profile plots of presynaptic (cyan) and postsynaptic (green) cytoplasm. Profiles were aligned to the lipid membrane peak of the PreSM and PoSM (grey arrows). The average intensity profile of 9 synapse tomograms is shown in black.

(B) Schematic showing cryoCLEM and cryoET workflow using thin vitreous sections from forebrains of adult $P s d 95^{G F P / G F P}$ knockin mice to determine the in-tissue architecture of glutamatergic synapses. Mice are culled and dissected. $100 \mu \mathrm{m}$ acute slices are collected, from which $2 \mathrm{~mm}$ diameter biopsy of cortex was high-pressure frozen. $150 \mathrm{~nm}$ thin cryo-sections were cut from vitrified tissue and attached to cryoEM grid for cryoCLEM and cryoET.

(C) Virtual slice through cryo-electron tomogram of PSD95-GFP containing synapse within thin vitreous cryo-section of adult mouse cortex. Cyan, PreSM. Green, PoSM. Scalebar, $50 \mathrm{~nm}$.

(D) Same as A for data obtained from vitreous cryo-sections from 5 synapse tomograms. Average intensity profile shown in black.

(E) Prevalence of membrane bound organelles within the PreSM (cyan) and PoSM (green) compartments identified in tomograms of forebrain PSD95-GFP containing synapses from 5 adult $P s d 95^{G F P / G F P}$ mice. Error bars, SEM.

(F) Prevalence of structural classes of membrane-bound organelles, including polyhedral vesicles, flat/tubular membrane compartments, large spheroidal membrane compartments $(>60 \mathrm{~nm}$ diameter), multivesicular bodies, and polyhedral vesicles within the PreSM (cyan) and PoSM (green) compartments of PSD95-containing forebrain synapses from 5 adult Psd95 ${ }^{\text {GFP/GFP }}$ mice. Error bars, SEM.

(G-I) Organelles in PSD95-containing synapse shown top, as a virtual slice through tomographic map and bottom, 3D segmentation. Cyan, PreSM. Green, PoSM. Red, green and blue arrows indicate $x$-, $y$-, and $z$-axis of tomogram, respectively. Scale bar, $100 \mathrm{~nm}$.

(G) Flat/tubular membrane compartment and large spheroidal membrane compartment pseudocoloured orange and red, respectively.

(H) Multivesicular bodies pseudo-coloured orange.

(I) Polyhedral membrane vesicle pseudo-coloured orange 


\section{Figure 3}

A
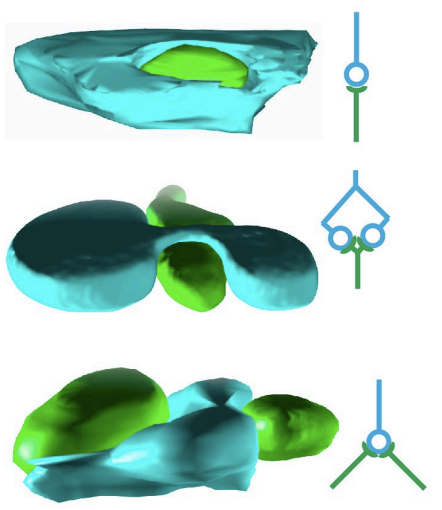

B

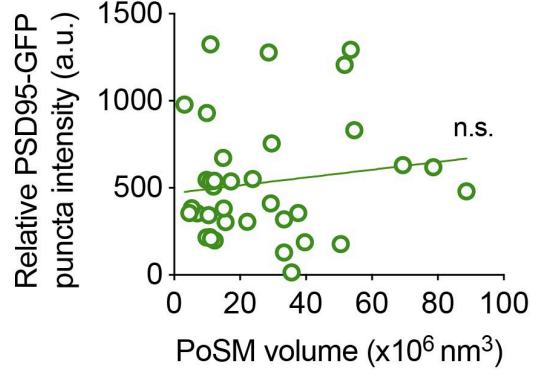

C

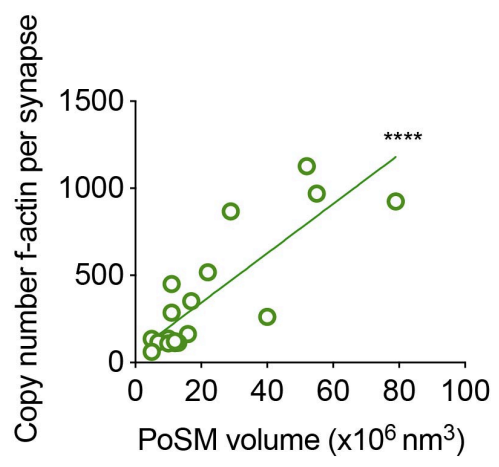

D

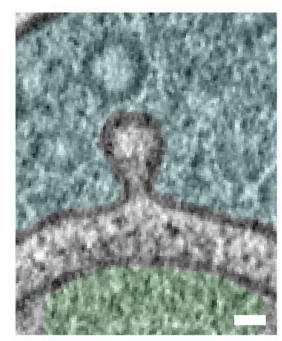

G

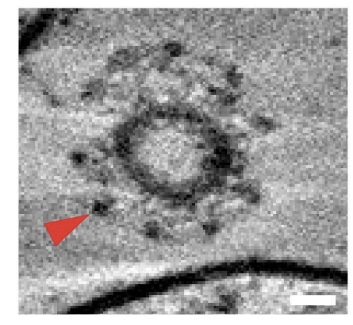

E

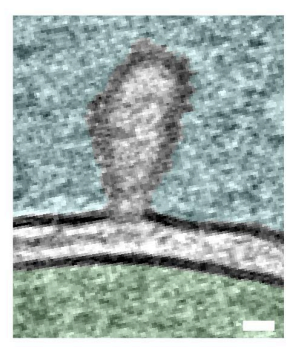

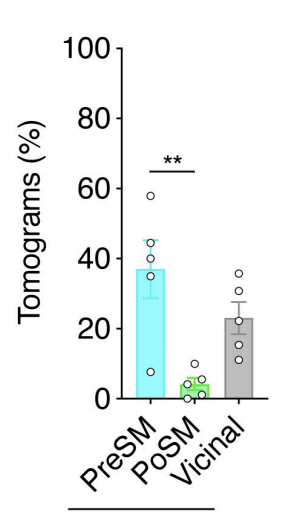

Membrane fission/ fusion intermediate
$\mathbf{F}$

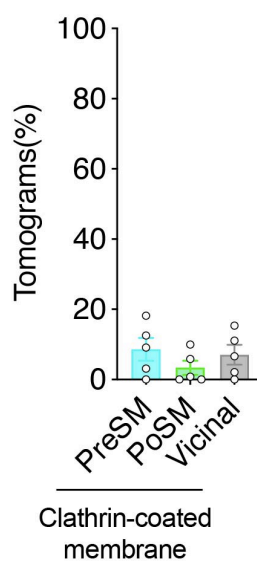

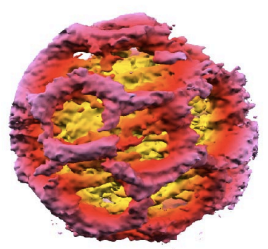

H

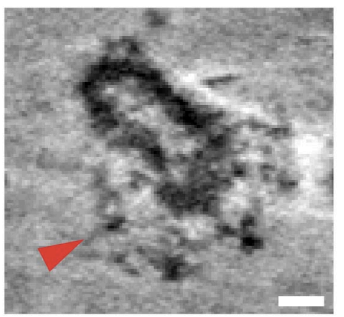

I

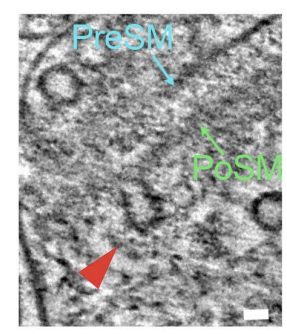

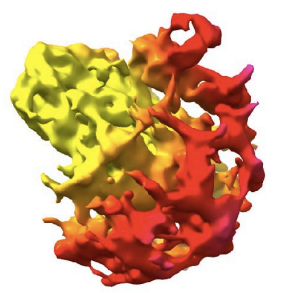

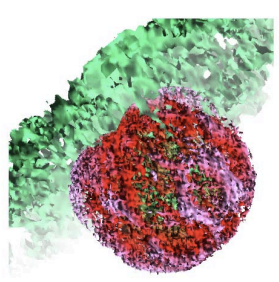


Figure 3. Topology and membrane remodeling within glutamatergic synapses.

(A) 3D segmentation (left) and schematic (right) of PSD95-GFP-containing synapses showing various topologies of connectivity. Top, unimodal single input and single output. Middle, bifurcated synapse with single input and output. Bottom, divergent synapse with single input and two outputs. Cyan, PreSM input. Green, PoSM output.

(B) Plot of fluorescence intensity (from cryoFM of PSD95-GFP) versus PoSM volume suggesting that there is no correlation between apparent amount of PSD95-GFP and PoSM volume. Linear regression (green line) with Pearson's $\mathrm{r}=0.14, \mathrm{P}=0.41$.

(C) Plot showing the copy number of F-actin cytoskeletal elements in PoSM compartment versus PoSM volume of each synapse. The copy number of F-actin cytoskeleton was estimated from 3D segmented models of $7 \mathrm{~nm}$ filaments within the tomographic map. Linear regression (green line) with Pearson's r $=0.83, \mathrm{P}<0.0001$.

(D-I) Snapshots of membrane remodeling within glutamatergic synapses.

(D) Virtual slices of tomographic map of synapses with intermediates of vesicle fusion/fission on the presynaptic side of the cleft of a PSD95-GFP containing synapse. Left, small intermediate. Right, Large intermediate. Scale bar, $20 \mathrm{~nm}$.

(E) Prevalence of membrane fission/fusion intermediates within PreSM, PoSM, and non-synaptic membranes (vicinal) from tomograms of 5 adult $P s d 95^{G F P / G F P}$ mice. Error bars, SEM.

(F) Same as E but for clathrin-coated membrane.

(G) Top, virtual slice through tomographic map of a clathrin-coated (red arrowhead) synaptic vesicle and bottom, a 3D tomographic density map for the region shown on top. Clathrin cage and membrane is shown with red and yellow voxels respectively. Scale bar, $20 \mathrm{~nm}$.

(H) Top, virtual slice through tomographic map of a clathrin-coat (red arrowhead) encapsulating part of an internal membrane within the PreSM compartment of a PSD95-GFP containing synapse and shown bottom, a masked 3D tomographic map. Clathrin cage and membrane is shown with red and yellow voxels, respectively. Scale bar, $20 \mathrm{~nm}$.

(I) Top, virtual slice, and bottom, masked 3D tomographic map showing clathrin-coated endocytic pit (red arrowhead) within the cleft of a PSD95-GFP containing PoSM. Clathrin cage and membrane is shown with red and green voxels, respectively. Scale bar, $20 \mathrm{~nm}$. 
Figure 4

A

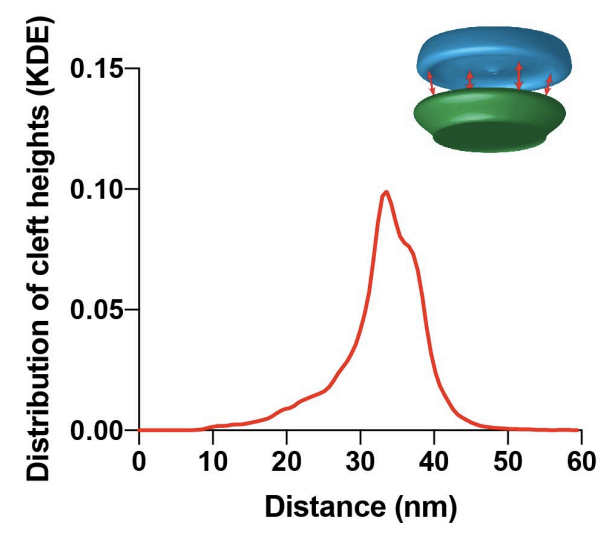

B

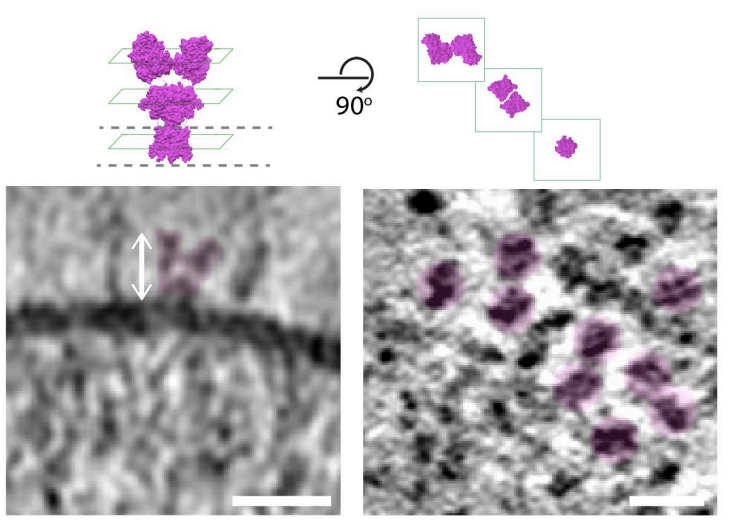

C
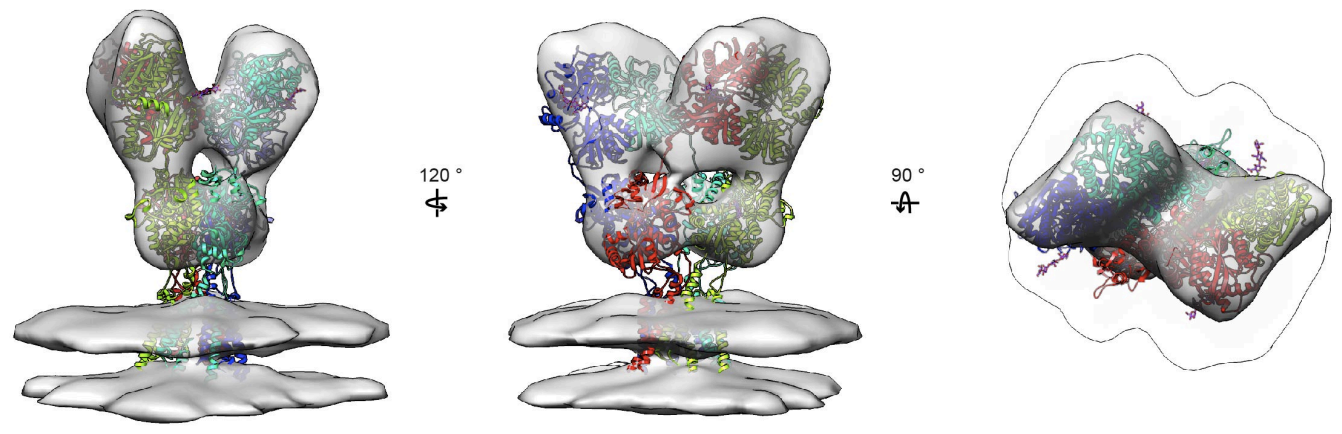

D

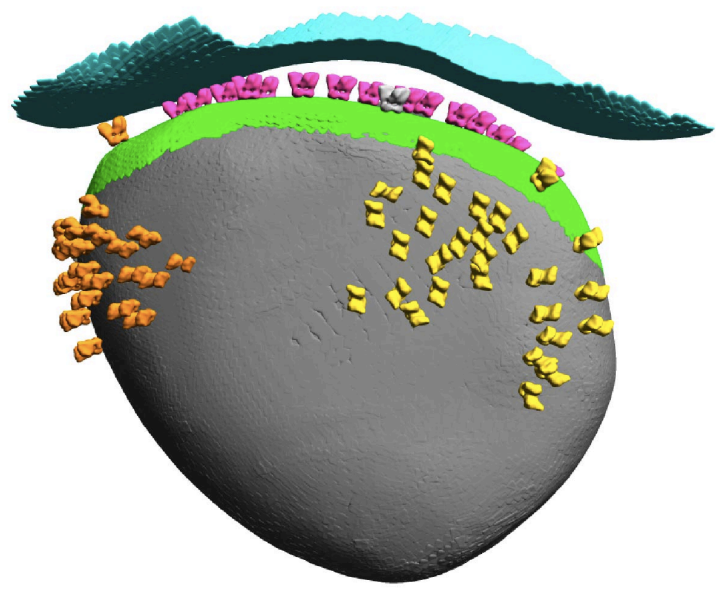

E

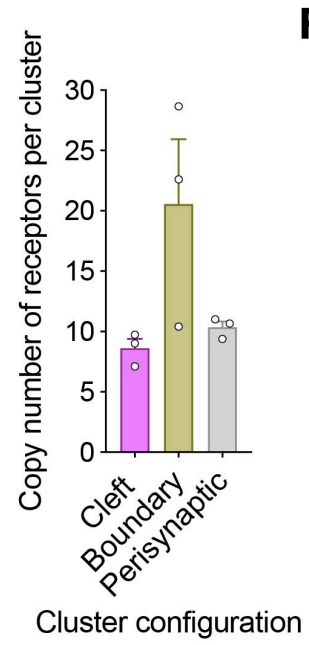

F

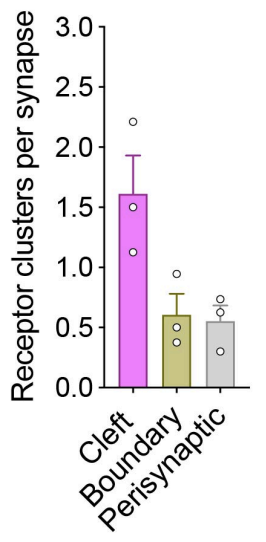

Cluster configuration 
Figure 4. Structural variables of synaptic strength.

(A) Distribution of cleft height distances of all synapses is shown as a kernel density estimation (KDE). Inset, schematic depicting nearest neighbour method for defining the synaptic cleft between PreSM (cyan) and PoSM (green) compartments. See Fig. S4A and B showing 3D segmented model of synaptic cleft and distribution of cleft heights for each individual synapse, respectively.

(B) Top left, 'side' view of atomic structure of AMPA subtype of ionotropic glutamate receptor (PDB ID: 3kg2). Dashed line, boundary of transmembrane domain. Top right, 'top' views of distal amino-terminal domain layer, proximal ligand-binding domain layer, and transmembrane domain layer. Bottom left, virtual slice of tomographic map of PSD95-GFP containing membrane oriented approximately parallel to the electron beam. Putative ionotropic glutamate receptor pseudocoloured in magenta. Bottom right, virtual slice through tomographic volume of PSD95-GFP containing membrane oriented approximately orthogonal to the electron beam. Multiple putative ionotropic glutamate receptor pseudo-coloured in magenta. Scale bar, $20 \mathrm{~nm}$.

(C) In situ structure of ionotropic glutamate receptors within PSD95-GFP containing PoSM determined by subtomogram averaging of 2,368 subvolumes. Atomic model of AMPA receptor (GluA2, PDB: $3 \mathrm{~kg} 2$ ) docked in the determined map. Subunits of the model are coloured in red, yellow, cyan and purple.

(D) Cluster analysis of identified ionotropic glutamate receptors depicted as a 3D model of the postsynaptic compartment with ionotropic glutamate receptors in the orientation and position determined by sub-tomogram averaging. Each cluster is coloured differently: magenta, orange, and yellow. Presynaptic and postsynaptic cleft membrane are shown in cyan and green, respectively. Postsynaptic membrane outside of the cleft is shown in grey.

(E) Receptor number per cluster for three different cluster locations: in the cleft (magenta), partly inside and outside the cleft (boundary, yellow), and completely outside the cleft (perisynaptic, grey) from tomograms of 3 adult $P s d 95^{\text {GFP/GFP }}$ mice.

(F) Prevalence of receptor clusters for three different cluster locations: in the cleft (magenta), partly inside and outside the cleft (boundary, yellow), and completely outside the cleft (perisynaptic, grey) from tomograms of 3 adult $P s d 95^{G F P / G F P}$ mice. 


\section{Methods}

\section{Mouse genetics and breeding}

Animals were treated in accordance with UK Animal Scientific Procedures Act (1986) and NIH guidelines. The generation and characterization of the PSD95-GFP knockin mouse $\left(P s d 95^{G F P}\right)$ is described in $(12,13)$. The endogenous PSD95 protein levels, assembly into supercomplexes, anatomical localization, developmental timing of expression and electrophysiological function in the hippocampus CA1-CA3 synapses in $P s d 95^{G F P}$ mice are indistinguishable from WT mice (2).

\section{Ultra-fresh synapse preparation}

Samples were prepared from adult (P65-P100) male Psd95 ${ }^{\text {GFP/GFP }}$ knockin mice. Sample preparation from culling to cryopreservation of each mouse was less than 2 minutes. Mice were culled by cervical dislocation. Forebrain, including cortex and hippocampus, were removed (in $<40 \mathrm{~s}$ ) and were homogenized ( $<20 \mathrm{~s}$ ) by applying 12 strokes of a Teflon-glass pestle and mortar containing $5 \mathrm{ml}$ ice-cold carboxygenated $\left(5 \% \mathrm{CO}_{2}, 95 \% \mathrm{O}_{2}\right)$ artificial cerebrospinal fluid (ACSF; $125 \mathrm{mM} \mathrm{NaCl}, 25 \mathrm{mM} \mathrm{KCl}, 25 \mathrm{mM} \mathrm{NHCO}_{3}, 25 \mathrm{mM}$ glucose, $2.5 \mathrm{mM} \mathrm{KCl}, 2 \mathrm{mM} \mathrm{CaCl} 2,1.25$ $\mathrm{mM} \mathrm{NaH} 2 \mathrm{PO}_{4}, 1 \mathrm{mM} \mathrm{MgCl}$; Osmolality: $310 \mathrm{mOsM} / \mathrm{L}$ ). 1 ul homogenate was diluted into $100 \mu \mathrm{l}$ ice-cold ACSF containing 1:6 BSA-coated $10 \mathrm{~nm}$ colloidal gold (BBA; used as a fiducial maker) $(<10$ s). 3 ul sample was applied onto a glow-discharged 1.2/1.3 carbon foil supported by 300-mesh $\mathrm{Au}$ or $\mathrm{Cu}$ grid held at $4{ }^{\circ} \mathrm{C}, 100 \%$ humidity. Excess sample was blotted from the non-foil side of the grid for $\sim 4 \mathrm{~s}$ with Whatman paper (No. 1) before cryopreserving by plunge freezing in liquid ethane held at $-180^{\circ} \mathrm{C}(49)$. Forebrain samples prepared from 5 mice prepared on 6 grids that used for data collection.

\section{High pressure freezing and cryo-ultramicrotomy}

Samples were prepared from adult (P65-P100) male Psd95 $5^{E G F P / E G F P}$ knockin mice. Mice were culled by cervical dislocation. Forebrain, including cortex and hippocampus, were removed and $100 \mu \mathrm{m}$ acute slices were prepared using a Leica VT1200S vibratome at $0.5-2^{\circ} \mathrm{C}$. Slices were recovered in carboxygenated ACSF perfused with carboxygen at room temperature for 45 minutes before being transferred to carboxygenated ASCF supplemented with 20\% dextran 40,000 from which $2 \mathrm{~mm}$ diameter biopsy samples of the cortex were collected using tissue punch and highpressure freezing samples in $3 \mathrm{~mm}$ carriers using a Leica EM ICE. Vitreous tissue was cut into 150 nm sections using a Leica FC7 cryo-ultramicrotome with a CEMOVIS diamond knife (Diatome) 
and mounted onto cryoEM grids at $-150^{\circ} \mathrm{C}$.

\section{Cryogenic correlated light and electron microscopy}

Cryogenic fluorescence microscopy (cryoFM) was performed on the Leica EM cryoCLEM system with a HCX PL APO 50x cryo-objective with NA = 0.9 (Leica Microsystems), an Orca Flash 4.0 V2 sCMOS camera (Hamamatsu Photonics), a Sola Light Engine (Lumencor) and the following filters (Leica Microsystems): green (L5; excitation 480/40, dichroic 505, emission 527/30), red (N21; excitation 515-560, dichroic 580, emission LP 590), and far-red (Y5; excitation 620/60, dichroic 660, emission 700/75). During imaging, the humidity of the room was controlled to 20$25 \%$ and the microscope stage was cooled to $-195^{\circ} \mathrm{C}$. Ice thickness was assessed with a brightfield image and L5 filter. Regions of the grid with thin ice ( $\sim 50 \%)$ were imaged sequentially with the following channel settings: $0.4 \mathrm{~s}$ exposure $3 \mathrm{~s}$ exposure, $30 \%$ intensity in green channel; $40 \mathrm{~ms}$ exposure, intensity $11 \%$ in BF channel; $0.4 \mathrm{~s}$ exposure, $30 \%$ intensity in red channel, $0.4 \mathrm{~s}$ exposure, $30 \%$ intensity in far-red channel. $0.3 \mu \mathrm{m}$ separated $\mathrm{z}$-stack of images was collected for each channel over a 5-20 $\mu \mathrm{m}$ focal range. Images were processed in Fiji. The location of PSD95-GFP was evident as puncta of varying brightness that were exclusively in the green channel. The occasional autofluorescent spot was identifiable by fluorescence across multiple channels, including green and red channels. Grid squares were selected for cryoCLEM that contained PSD95-GFP puncta within the holes of the holey carbon grid.

\section{Cryogenic electron microscopy and tomography}

CryoEM and CryoET data collection was performed on a Titan Krios microscope (FEI) fitted with a Quantum energy filter (slit width $20 \mathrm{eV}$ ) and a K2 direct electron detector (Gatan) running in counting mode with a dose rate of $\sim 4 \mathrm{e}^{-} / \mathrm{pixel} / \mathrm{second}$ at the detector level during tilt series acquisition. Intermediate magnification EM maps of the selected grid squares were acquired at a pixel size of $5.1 \mathrm{~nm}$. Using these intermediate magnification maps of each grid square and the corresponding cryoFM image, the location of PSD95-GFP puncta was manually estimated; performing computational alignment of cryoFM and cryoEM images before tomogram acquisition was not necessary.

Tomographic tilt series of the cryoFM-correlated PSD95-GFP locations were collected between $\pm 60^{\circ}$ using a grouped dose-symmetric tilt scheme (50) in serialEM (51) and a phase plate (52) pre-conditioned for each tomogram. Groups of 3 images with $2^{\circ}$ increments were collected. Images with a $2 \mathrm{~s}$ exposure with $0.8-1.25 \mu \mathrm{m}$ nominal defocus, at a dose rate of $\sim 0.5 \mathrm{e}^{-} / \AA / \mathrm{s}$ were 
collected as $8 \times 0.25 \mathrm{~s}$ fractions, giving a total dose $\sim 60 \mathrm{e}^{-} / \AA \AA$ over the entire tilt series at a calibrated pixel size of $2.89 \AA$. Tomograms of thin vitreous sections were collected with the same protocol but replacing the phase plate with $100 \mu \mathrm{m}$ objective aperture and acquiring tilt series images at 5$6 \mu \mathrm{m}$ nominal defocus with a nominal pixel size of $3.42 \AA$.

\section{CryoCLEM image processing}

Computational correlation between the cryoFM and cryoEM images was conducted using custom Matlab (Mathworks) scripts as described previously $(53,54)$. The centre points of at least 10 holes in the holey-carbon foil around each PSD95 position were used as fiducial markers to align the green channel cryoFM image to a montaged intermediate magnification EM image covering the whole grid square. All GFP puncta correlated within membrane-bound compartments with an apparent presynaptic membrane attached to the postsynaptic membrane. The identity of presynaptic membranes was confirmed by the presence of numerous synaptic vesicles.

To quantify relative amount of PSD95 in each synapse, PSD95-EGFP puncta in cryoFM images were segmented using the watershed algorithm in ImageJ, from which the pixel intensities of each puncta were integrated.

\section{Tomogram reconstruction}

Frames were aligned and tomograms were reconstructed using 10-20 tracked $10 \mathrm{~nm}$ fiducial gold markers in IMOD (55). Tomograms of synapses in thin vitreous sections were denoised using JANNI in crYOLO (50) and aligned by patch tracking. Tomograms used for figures, particle picking, annotation of macromolecular constituents and molecular density analysis were generated with 5 iterations of SIRT and binned to a $11.94 \AA$ (binned 4x). Weighted back-projection tomograms (binned $4 \mathrm{x}$ and $2 \mathrm{x}$ ) were used for subtomogram averaging (see below).

\section{Annotation and analysis of macromolecular constituents in tomograms}

Annotation was performed blind by two curators. First, each curator annotated all SIRT reconstructed tomograms independently. Next, a third curator inspected and certified each annotation (Table S1). The PoSM was identified using PSD95-GFP cryoCLEM (see above). The PreSM was identified as the membrane compartment attached to the PoSM via transsynaptic adhesion proteins and containing numerous synaptic vesicles (25-35 nm diameter).

Molecular density analysis of the PreSM and PoSM compartments was carried out using 30 voxel 
intensity line profiles of the presynaptic and postsynaptic membrane measured in Fiji. For each synapse 30 measurements were taken sampling in multiple positions within the cleft. Profiles were averaged and background subtracted to estimate the relative density of molecular crowding within the PreSM and PoSM compartments of each synapse.

Organelles, intermediates of membrane fission or fusion, and identifiable macromolecules were annotated within the presynaptic, postsynaptic and non-synaptic (vicinal) compartments within tomogram dataset (Table S1), which were classified as follows: 1) Flat/tubular membrane compartments: Flat membrane tubules $12-20 \mathrm{~nm}$ wide that twists along the tubule axis by at least $180^{\circ}$. 2) Large spheroidal membrane compartments: Vesicles with a diameter greater than $60 \mathrm{~nm}$. 3) Multivesicular bodies: Membranous compartments containing at least one internal vesicle. 4) Polyhedral vesicles: Membranous compartment composed of flat surfaces related by $\leq 90^{\circ}$ angle. 6 ) Dense-cored vesicles. 7) Fission/fusion intermediates: hemifusion intermediates, protein-coated membrane invaginations. 8) Clathrin-coated intermediates: invaginations with a protein coat containing pentagonal or hexagonal openings. 9) Mitochondria: double membrane with cristae and electron-dense matrix. 10) Mitochondrial intermediates: mitochondria with budding outer membrane or putative mitochondrial fragments wrapped by two membrane bilayers. 11) Ribosomes: 25-35 nm electron dense particles composed of small and large subunits. 12) F-actin: $7 \mathrm{~nm}$ helical filaments. 13) Microtubules: $25 \mathrm{~nm}$ filaments. 14) Cargo-loaded microtubules: vesicles attached to microtubules via $25-30 \mathrm{~nm}$ protein tethers.

\section{Quantitative analysis of the PreSM, PoSM, and synaptic cleft}

Each membrane was segmented in Dynamo (57), generating 4,000-20,000 coordinates for each membrane. To refine the contouring of the membrane, subtomograms of the membrane were refined against an average. The synaptic cleft was defined computationally using custom Matlabbased script that identifies the nearest neighbour coordinates between PreSM and PoSM, and vice versa.

\section{Subtomogram averaging}

Subtomogram alignment and averaging was performed using Matlab-based scripts derived from the TOM (58) and Av3 (59) toolboxes, essentially as described in (60).

Coordinates of putative ionotropic glutamate receptors were manually picked in UCSF Chimera using two times binned tomograms with an effective voxel size of $11.94 \AA$. All membrane proteins 
with a long-axis extending $\sim 14 \mathrm{~nm}$ out of the membrane and an apparent two-fold symmetry axis were picked. The orientation of the normal vector to the membrane surface was determined to define the initial orientation of each subtomogram. The Euler angle defining the final in-plane rotation angle (phi in the av3 annotation) was randomized. The full set of subtomogram positions was split into two independent half sets one for all odd and one for all even subtomogram numbers. The two half sets were processed independently. For initial alignment and averaging, subtomograms with a box size of $64 \times 64 \times 64$ voxels $\left(\sim 76.4 \times 76.4 \times 76.4 \mathrm{~nm}^{3}\right)$ were extracted from the respective tomograms and averaged. The initial average shows a long, thin stalk. After one iteration of alignment using a low pass filter of $30 \AA$ and a complete in-plane angular search, two fold symmetry was applied for further iterations. During all steps of alignment and averaging, the missing wedge was modelled as the sum of the amplitude spectra determined from 100 randomly seeded noise positions for each tomogram. After 6 iterations subtomograms with a box size of 96x96x96 voxels $\left(\sim 53.7 \times 53.7 \times 53.7 \mathrm{~nm}^{3}\right)$ were re-extracted from tomograms with a voxel size of $5.97 \AA$. Subtomogram positions were reprojected onto the respective tomogram for visual inspection, and mis-aligned subtomogram positions as well as subtomograms that had converged to the same position were removed, reducing the final number of subtomograms to 2368 subtomograms (odd=1182 and even=1186). The two averages were then further refined for 4 iterations using a cylindrical mask tightly fit around the obtained structure and including the membrane and subsequently for 6 more iterations using a cylindrical mask excluding the membrane. To evaluate the final average and assess the resolution obtained, the averages from the two half sets were aligned to each other and the FSC was calculated as described in (61) indicating a final resolution of $25 \AA \AA$ (Fig. S5A). The previously reported atomic coordinates of an AMPA subtype ionotropic glutamate receptor (42)(PDB: $3 \mathrm{KG} 2)$ were fitted as a rigid body into the final subtomogram averaging map using the fit in map tool in UCSF Chimera (62).

\section{Cluster analysis}

Clustering analysis of glutamate receptors was performed using the DBSCAN algorithm (minimal cluster size 4 and maximal distance between receptors of $70 \mathrm{~nm}$ ) where the distances between pairs of receptors were defined as the shortest distance between their projections on the surface of the membrane. The mesh associated to the surface was estimated using the "MyOpenCrust" implementation of the crust meshing method and simplified using the reducepatch from Matlab. The distances between the projected coordinates were computed using the Dijkstra algorithm on the graph associated to the mesh. 